OPEN

SUBJECT AREAS:

COMPLEX NETWORKS

SOCIAL EVOLUTION

Received

31 December 2013

Accepted

14 March 2014

Published

4 April 2014

Correspondence and requests for materials should be addressed to X.L. (lix@fudan.edu.cn)

* These authors contributed equally to this work.

\section{Evolutionary Origin of Asymptotically Stable Consensus}

\author{
Chang-Bing Tang ${ }^{1 *}$, Bin Wu ${ }^{2 *}$, Jian-Bo Wang ${ }^{1} \&$ Xiang $\mathrm{Li}^{1}$
}

\begin{abstract}
'Adaptive Networks and Control Lab, Department of Electronic Engineering, Fudan University, Shanghai 200433, PR China,
${ }^{2}$ Research Group for Evolutionary Theory, Max-Planck-Institute for Evolutionary Biology, August-Thienemann-Straße 2, 24306 Plön, Germany.
\end{abstract}

Consensus is widely observed in nature as well as in society. Up to now, many works have focused on what kind of (and how) isolated single structures lead to consensus, while the dynamics of consensus in interdependent populations remains unclear, although interactive structures are everywhere. For such consensus in interdependent populations, we refer that the fraction of population adopting a specified strategy is the same across different interactive structures. A two-strategy game as a conflict is adopted to explore how natural selection affects the consensus in such interdependent populations. It is shown that when selection is absent, all the consensus states are stable, but none are evolutionarily stable. In other words, the final consensus state can go back and forth from one to another. When selection is present, there is only a small number of stable consensus state which are evolutionarily stable. Our study highlights the importance of evolution on stabilizing consensus in interdependent populations. 


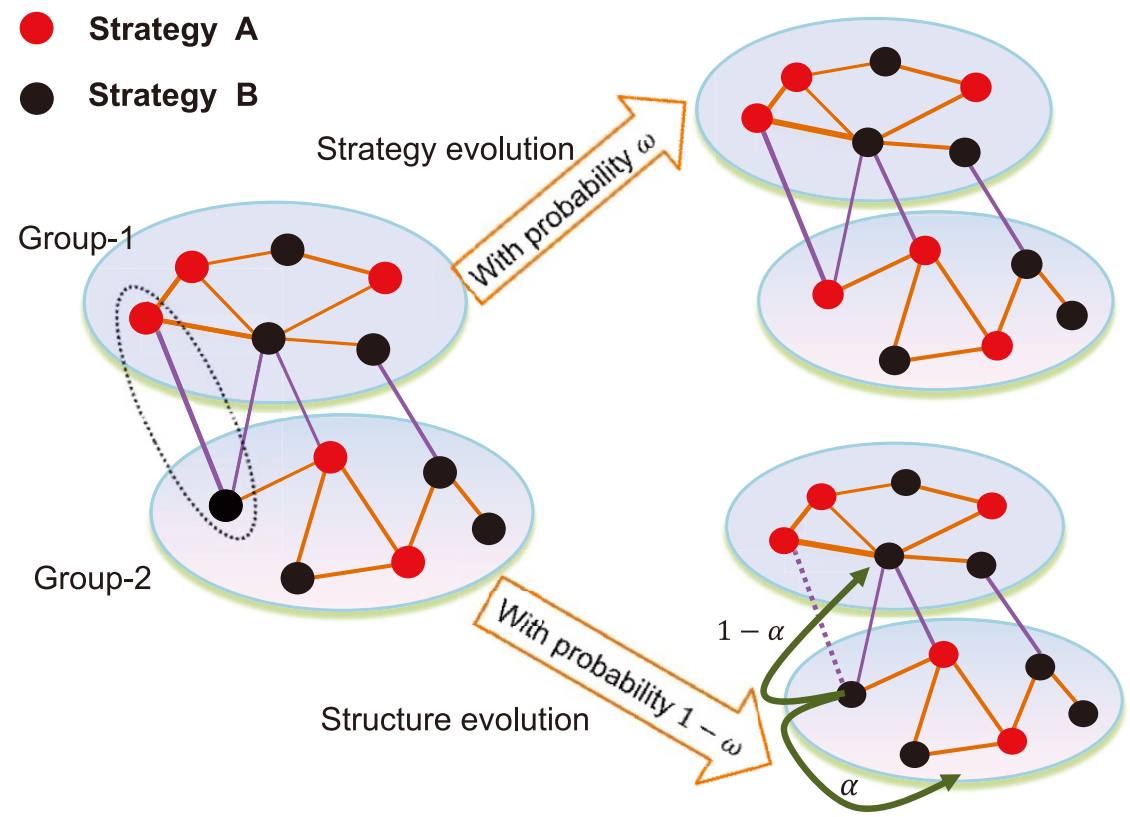

Figure 1 The co-evolution of strategy updating and structure switching. Each node denotes one of two types of players, $A$ and $B$, arranged in two interactive groups referred as Group-1 and Group-2, respectively. At each time step, the strategy updating happens with probability $\omega$; the evolution of structures happens with probability $1-\omega$. For rewiring partnerships during the structure evolution process, we choose neighbours in its own group with probability $\alpha$, and choose neighbours in the other group with probability $1-\alpha$.

\section{Model}

Micro-update rules in interdependent populations. Individuals are located at nodes in interdependent populations, consisting of two interactive groups referred as Group-1 and Group-2 (See Figure 1). Note that this set-up is akin to the structure of interdependent networks, in the sense that the success of one node in a given group not only depends on the nodes in the same group, but also replies on the state of other nodes in other groups. Group- $i$ is of size $N_{i}(i=1,2)$, leading to the size of entire population $N=N_{1}+N_{2}$. Initially, the average degree of Group- $i$ is denoted as $L_{i}=2 H_{i} / N_{i}$, where $H_{i}$ is the total number of links in Group-i. Denote the number of links connecting two nodes in Group- $i$ as $H_{i i}(i=1,2)$, while the number of links connecting two nodes between two groups as $H_{12}$ (or $H_{21}$ ). Then, the total number of links is $H=H_{12}+H_{11}+H_{22}$.

Each player is either of strategy $A$ or $B$, denoted by $(1,0)^{T}$ and $(0,1)^{T}$, respectively. Here, a strategy represents an opinion/a behavior adopted by players. When two $A$ (player with strategy $A$ ) meet, each player gets payoff $a$; when two $B$ (player with strategy $B$ ) meet, each player gets payoff $d$. When $A$ meets $B, A$ gets payoff $b$ while $B$ gets payoff $c$, which leads to a payoff matrix $M$ describing the local pairwise interaction as follows:

$$
\begin{aligned}
& \text { A } B \\
& { }_{B}^{A}\left(\begin{array}{ll}
a & b \\
c & d
\end{array}\right) .
\end{aligned}
$$

Without loss of generality, we assume that all payoff entries are positive. The payoff of each player is obtained by playing with all its immediate neighbors.

We consider the co-evolution of strategy and interdependent populations ${ }^{4-45}$. In fact, not only strategy but also social interactions between individuals are under the pressure of evolution, which leads to the entangled dynamics of strategy and structures, and such coevolution is an important source of dynamical complexity ${ }^{46}$.

At each time step, with certain probability $\omega$, strategy evolution happens; otherwise, the evolution of structures occurs. Here, probability $\omega$ governs the dynamical time-scale of these two updating rules. When $\omega=1$, the strategy evolves while population structure never changes, which has been extensively addressed ${ }^{20-24}$. When $\omega=0$, no strategy evolution occurs while the topology evolves all the time. In this case, the frequency of a specified strategy in any group will never change, thus the population-level consensus in two interactive-groups cannot takes place, unless it is in the consensus state initially. The rules of strategy updating and structure switching are listed as follows:

Strategy evolution. We adopt the Fermi update dynamics ${ }^{47,48}$ to describe the strategy evolution, where individuals update their strategies by imitating the strategy of more successful individuals. At each time step (during the strategy evolution), a player is selected randomly from the whole population, namely $h$ with payoff $P_{h}$, then a neighbor of $h$ is selected randomly, namely $g$ with payoff $P_{g}$. Here, $P_{h}=\sum_{i=1}^{2} P_{i, h}$ is the payoff of player $h\left(P_{i, h}\right.$ is the payoff of player $h$ obtained from Group-i). Then, the strategy of player $h$ replaces that of player $g$ with probability $p=\left[1+e^{-\beta\left(P_{h}-P_{g}\right)}\right]^{-1}$, where $\beta \geq 0$ is the intensity of imitation, measuring how strong the payoff difference influences individuals' decision making.

Structure evolution. Each link is assigned a number, $l \in\{1,2, \cdots, H\}$, as its name. To characterize the dynamics of population structure with various kinds of relationships, where players leave or break interactions when they dissatisfy with the current situation, we introduce $k_{X Y}$ as the probability with which a $X Y$-type link breaks. We describe the structure evolution in detail as below:

Step 1. At each time $t$ (during the structure evolution), a link $l^{t}$ of type $X_{i} Y_{j}$ is selected from the interdependent populations at random $(X, Y$ $\in\{A, B\}, i, j \in\{1,2\})$.

Step 2. With probability $1-k_{X Y}$, the selected link $l^{t}$ remains unchanged, denoted as $l^{t+1}=l^{t}$. With probability $k_{X Y}$, the selected link breaks.

Step 3. If the link is broken, one node is selected randomly from the two ends, then the selected player tries to find another player to newly 
connect with. With probability $\alpha$, the neighbor is only selected within the same group as its friend-making candidate; otherwise, the potential neighbor is chosen from the other group. Denote this new link as $l^{t+1}$.

Here, $\alpha$ is the intra-group attaching bias between two interactive groups, reflecting the propensity to rewire neighbors within the same group; and $k_{X Y}$ is time-invariant, reflecting the structure effect of linking dynamics. Note that the inverse of $k_{X Y}$ can be regarded as the average duration time between strategies $X$ and $Y$, namely $k_{X Y}$ is a measurement of the duration of $X Y$ link. Furthermore, the total number of links $H$ is fixed in the co-evolution process ${ }^{41,43,49,50}$, which implies a limited resource environment. The implementation of the co-evolution of strategy and structure scheme is depicted as shown in Figure 1.

Macro-dynamics in interdependent populations. Consider the fast partnership rewiring process, i.e., $\omega \ll 1$, which corresponds to the case that players are much more reluctant to change strategies than to adjust their interactions ${ }^{49-52}$. In this case, the structure evolution of the interdependent populations obeys a stationary distribution when the strategy evolution occurs (See Section 2 in SI). Moreover, the average fitness of players is determined by the stationary distribution of the structure evolution. Therefore, the average fitness of strategies $A$ and $B$ in Group- $i$ are

$$
\left\{\begin{array}{l}
f_{i, A}=\frac{\mathcal{S}}{N_{i}}\left[\alpha e_{1} M^{\prime} x_{i}+(1-\alpha) e_{1} M^{\prime} x_{j}\right] \\
f_{i, B}=\frac{\mathcal{S}}{N_{i}}\left[\alpha e_{2} M^{\prime} x_{i}+(1-\alpha) e_{2} M^{\prime} x_{j}\right]
\end{array}\right.
$$

where $\mathcal{S}=H \cdot h\left(x_{i}, x_{j}\right), e_{1}=(1,0), e_{2}=(0,1), x_{i}=\left(x_{i, A}, x_{i, B}\right)^{T}\left(x_{i, A}\right.$ represents the fraction of strategy $A$ in Group-i), and

$$
M^{\prime}=\left(\begin{array}{cc}
\frac{a}{k_{A A}} & \frac{b}{k_{A B}} \\
\frac{c}{k_{A B}} & \frac{d}{k_{B B}}
\end{array}\right) .
$$

It is worth noting that matrix $M^{\prime}$ is a time-scaling modification of Eq. (1): every payoff entry is rescaled by the corresponding interaction time, i.e., the inverse of the breaking probability, based on what strategies the focal two players possess ${ }^{50}$. This transformation shows the complex dynamics incorporating both strategy and independent populations can be treated as a new game in a wellmixed population ${ }^{53}$. Besides, the first term in the bracket of Eq. (2) represents the payoff obtained via intra-group interactions, while the second term represents the payoff obtained via inter-group interactions. They are balanced by propensity $\alpha$. This simple equation analytically captures the basic idea of the model: each player's payoff is determined on not only the structure configuration it belongs to but also that of the other group.

For large populations, the stochastic process can be well depicted by a set of stochastic differential equations referred as Langevin dynamics ${ }^{54}$. According to Eq. (2), we obtain a differential equation governing the frequency of strategy $A$ in each group (See Section 3 in SI):

$$
\begin{aligned}
\dot{x}_{i, A}= & \frac{N_{i}^{2} \cdot x_{i, A} x_{i, B}}{\left(N_{1}+N_{2}\right)^{2}} \tanh \left(\beta \frac{f_{i, A}-f_{i, B}}{2}\right)+\frac{N_{i} N_{j} \cdot x_{i, B} x_{j, A}}{\left(N_{1}+N_{2}\right)^{2}} \\
& \frac{1}{1+e^{-\beta}\left(f_{j, A}-f_{i, B}\right)}-\frac{N_{i} N_{j} \cdot x_{i, A} x_{j, B}}{\left(N_{1}+N_{2}\right)^{2}} \frac{1}{1+e^{-\beta\left(f_{j, B}-f_{i, A}\right)}}
\end{aligned}
$$

Here $i, j \in\{1,2\}$, and $i \neq j$.

\section{Results}

Selection plays an important role in evolutionary process, which determines the direction of evolution. The absence of selective difference is called neutral selection $(\beta=0)$. When selection acts, the transition probability becomes payoff dependent, which can be further classified into constant and frequency dependent selections.
Generally, the probability of one strategy replaces another is fairly complicated under frequency dependent selection ${ }^{55}$. Fortunately, many results have been obtained with the assumption of weak selection, where perturbation analysis analytically facilitates the derivation $^{56,57}$. For non-weak selection, the approach of perturbation analysis does not work, and the result under weak selection can be violated $^{58}$.

Neutral selection: $\boldsymbol{\beta}=\mathbf{0}$. When $\beta=0$, the selection dynamics are neutral, in which the evolutionary direction is random and independent of the players' payoffs. In this case, Eq. (4) is simplified as

$$
\dot{x}_{i, A}=\frac{1}{2} \frac{N_{i} N_{j}}{\left(N_{i}+N_{j}\right)^{2}}\left(x_{j, A}-x_{i, A}\right)
$$

Note that the points in line $x_{2, A}=x_{1, A}$ are the equilibria of Eq. (5). The corresponding eigenvalues of the Jacobian matrix at $x_{2, A}=x_{1, A}$ are $\lambda_{1}=0$ and $\lambda_{2}=-2$. Thus, the line $x_{2, A}=x_{1, A}$ is always a stable manifold, and system (5) always reaches consensus. Furthermore, all the consensus states are stable, but none are evolutionarily stable, i.e., final consensus can go back and forth from one state to another (See Fig. 2).

Weak selection: $\boldsymbol{\beta} \ll \mathbf{1}$. When $\beta \ll 1$, the effects of payoff differences are small, such that the evolutionary dynamics mainly results from random fluctuations. This case of weak selection means one phenotype is slightly advantageous over another ${ }^{59}$. In evolutionary biology and population genetics, it is widely accepted that most mutations confer small selective difference ${ }^{60,61}$.

For simplicity, we assume $N_{1}=N_{2}$. We verify the general case of $N_{1} \neq N_{2}$ in numerical simulations (See Figs. 3-7). When $N_{1}=N_{2}$, Eq. (4) becomes

$$
\begin{aligned}
\dot{x}_{i, A} & =2 \kappa_{i} x_{i, A}\left(1-x_{i, A}\right)\left(c_{1} x_{i, A}+c_{2} x_{j, A}+c_{5}\right) \\
& +\frac{1}{8}\left(x_{j, A}-x_{i, A}\right) \\
& +\kappa_{i} x_{j, A}\left(1-x_{i, A}\right)\left(c_{3} x_{i, A}+c_{4} x_{j, A}+c_{5}\right) \\
& +\kappa_{i} x_{i, A}\left(1-x_{j, A}\right)\left(c_{4} x_{i, A}+c_{3} x_{j, A}+c_{5}\right)
\end{aligned}
$$

where $\kappa=\kappa_{1}=\kappa_{2}=\frac{\beta \mathcal{S}}{16 N_{1}}$ is constant, $c_{1}=\alpha\left(\frac{a}{k_{A A}}-\frac{b+c}{k_{A B}}+\frac{d}{k_{B B}}\right)$, $c_{2}=(1-\alpha)\left(\frac{a}{k_{A A}}-\frac{b+c}{k_{A B}}+\frac{d}{k_{B B}}\right), c_{3}=\frac{(1-\alpha) a}{k_{A A}}-\frac{(1-\alpha) b}{k_{A B}}-\frac{\alpha c}{k_{A B}}+$ $\left.\frac{\alpha d}{k_{B B}}, c_{4}=\frac{\alpha a}{k_{A A}}-\frac{\alpha b}{k_{A B}}-\frac{(1-\alpha) c}{k_{A B}}+\frac{(1-\alpha) d}{k_{B B}}\right)$, and $c_{5}=\frac{b}{k_{A B}}-\frac{d}{k_{B B}}$. Note that $\kappa$ only influences the time-scale with none effect on the long run evolutionary outcome.

From Eq. (6), we get three possible equilibria, i.e., $E_{1}=(0,0), E_{2}=$ $(1,1), E_{3}=\left(E_{3}^{*}, E_{3}^{*}\right) . E_{1}$ and $E_{2}$ are at the corners, which denote that system (6) is composed of all- $B$ and all- $A$, respectively. $E_{3}$ is an interior equilibrium, which means that system (6) consists of $A$ and $B$ players (See Section 4 in SI for details about the stability of each equilibrium). With different parameters $c_{i}(1 \leq i \leq 5)$, we further discuss the consensus for system (6) under weak selection.

Case 1: Consensus of the dominant-type game.

When $\frac{a}{k_{A A}}>\frac{c}{k_{A B}}$ and $\frac{b}{k_{A B}}>\frac{d}{k_{B B}}+\varepsilon\left(\varepsilon=\frac{1}{8 \kappa}\right)$, strategy $A$ dominates strategy $B$ in the interdependent populations, if $k_{A A}<k_{A B}<$ $k_{B B}$. This result indicates that all players are more likely to interact with strategy $A$. In this case, $E_{1}$ is an unstable equilibrium, and $E_{2}$ is a stable equilibrium, which implies that the set of all- $A\left(E_{2}\right)$ is the unique evolutionary stable state of this game. Let us take the prisoner's dilemma (PD) game as an example, in which strategies $A$ and $B$ denote defection and cooperation, respectively. The frequency of 


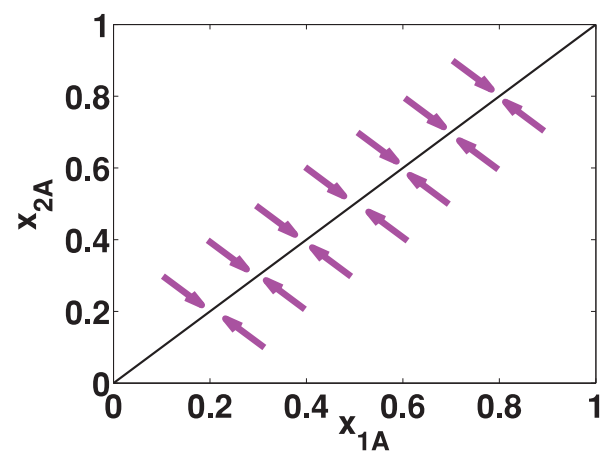

(a)

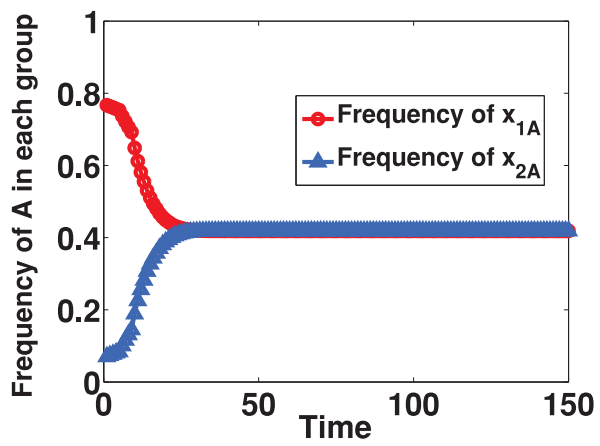

(b)

Figure $2 \mid$ System (5) reaches consensus under neutral selection $(\beta=0)$, with $N_{1}=150, N_{2}=100, L_{1}=6, L_{2}=9$. (a) Phase portrait of Eq. (5) where the direction of the velocity field is indicated by the arrows. The ultimate values of the frequency of strategy $A$ in Group- 1 and Group- 2 converge to the line $x_{2, A}=x_{1, A}$. (b) Simultaneous time-evolution of frequency of strategy $A$ in Group- 1 and Group-2. One of the consensus states is shown, where the initial frequency of strategy $A$ in Group- 1 and Group- 2 is selected randomly.

strategy $A$ in each group converges to the state of all- $A$, and system (6) reaches an asymptotically stable consensus state (See Fig. 3(a)).

When $\frac{a}{k_{A A}}+\varepsilon<\frac{c}{k_{A B}}$ and $\frac{b}{k_{A B}}<\frac{d}{k_{B B}}$, strategy $B$ dominates strategy $A$ in the interdependent populations, if $k_{A A}>k_{A B}>k_{B B}$. Therefore, all players are more likely to interact with strategy $B$. In this case, $E_{1}$ is a stable equilibrium, and $E_{2}$ is an unstable equilibrium. This situation is similar to that of the above discussion, which leads to the dynamics ending in the state of all- $B\left(E_{1}\right)$, and system (6) reaches an asymptotically stable consensus state, as shown in Fig. 3(b).

Therefore, when the interactions between players under the payoff matrix $M^{\prime}$ are of the dominant-type game, two interactive groups reach a homogeneous consensus, i.e., the stable consensus state consists of all- $A$, or all- $B$.

Case 2: Consensus of the coexisting-type game.

When

$$
\frac{a}{k_{A A}}+\varepsilon<\frac{c}{k_{A B}}, \quad \frac{b}{k_{A B}}>\frac{d}{k_{B B}}+\varepsilon,
$$

and

$\alpha<\alpha^{*}\left(\alpha^{*}=\frac{1}{2}-\frac{c_{1}}{16 \kappa c_{5}\left(c_{1}+\alpha c_{5}\right)}\right)$, both $E_{1}$ and $E_{2}$ are unstable, and $E_{3}$ is a saddle point, if $k_{A A}>k_{A B}$ and $k_{B B}>k_{A B}$. This implies that all players are inclined to interact with the opponents of opposed strategies as themselves'. In this case, the velocity field is out of order, and system (6) cannot reach a consensus state (See Fig. 4(a)). However, when the intra-group attaching bias $\alpha$ exceeds the critical value $\alpha^{*}, E_{3}$ becomes stable, and strategies $A$ and $B$ coexist in this interior stable equilibrium. The final state of the system converges to the asymptotically stable consensus state $E_{3}$ (See Fig. 4(b)). The corresponding representative model is the snowdrift game. Therefore, when interactions between players with the payoff matrix $M^{\prime}$ are of the coexisting-type game and the intra-group attaching bias exceeds the critical value $\alpha^{*}$, two interactive groups converge to a non-homogeneous consensus, i.e., the stable consensus state coexists with $A$ and $B$.

For populations with two static topology structures, the final state converges to the interior equilibrium $\left(-\frac{b-d}{a-b-c+d}\right.$, $\left.-\frac{b-d}{a-b-c+d}\right)^{33}$. For dynamic interdependent populations, the final dynamics ends at an interior equilibrium $E_{3}$ which results from the co-evolution of strategy and structure. In addition, although the intra-group attaching bias $\alpha$ does not affect the interior equilibrium $E_{3}$, it determines the stability of interior equilibrium. Only when $\alpha>\alpha^{*}$, the interior equilibrium is stable, i.e., a too strong rewiring propensity between two interactive groups (small $\alpha$ ) does not benefit the formation of an asymptotically stable consensus. To illustrate the effects of the intra-group attaching bias between two groups on consensus, we present the frequency of strategy $A$ in both groups as shown in Fig. 5. We observe that for $\alpha<\alpha^{*}$, system (6) cannot reach a consensus state (See Fig. 5(a) and Fig. 5(b)). When increasing the value of $\alpha$ until $\alpha>\alpha^{*}$, system (6) reaches an asymptotically stable consensus at interior equilibrium $E_{3}$. Interestingly, the increasing of $\alpha$ not only facilitates the formation of a consensus state in an

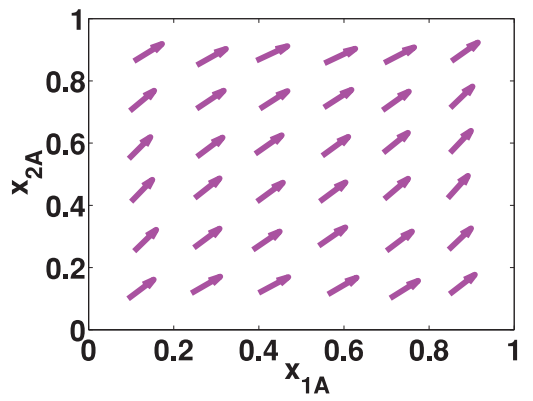

(a)

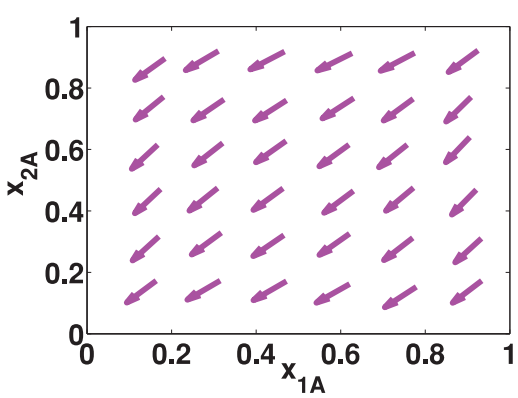

(b)

Figure $3 \mid$ Phase portrait of Eq. (6) under weak selection $(\boldsymbol{\beta}=\mathbf{0 . 0 1})$. The direction of the velocity field is indicated by arrows. We set $N_{1}=150, N_{2}=100$, $L_{1}=6, L_{2}=9, \omega=0.01$. (a) When $\frac{a}{k_{A A}}>\frac{c}{k_{A B}}$ and $\frac{b}{k_{A B}}>\frac{d}{k_{B B}}+\varepsilon\left(a=2, b=1, c=1.5, d=0, k_{A A}=0.4, k_{A B}=0.6\right.$ and $\left.k_{B B}=0.8\right)$, system (6) reaches an asymptotically stable consensus state of all- $A$. (b) When $\frac{a}{k_{A A}}+\varepsilon<\frac{c}{k_{A B}}$ and $\frac{b}{k_{A B}}<\frac{d}{k_{B B}}\left(a=1.5, b=0, c=2, d=1, k_{A A}=0.6, k_{A B}=0.4\right.$ and $\left.k_{B B}=0.8\right)$, system (6) reaches an asymptotically stable consensus state of all- $B$. 


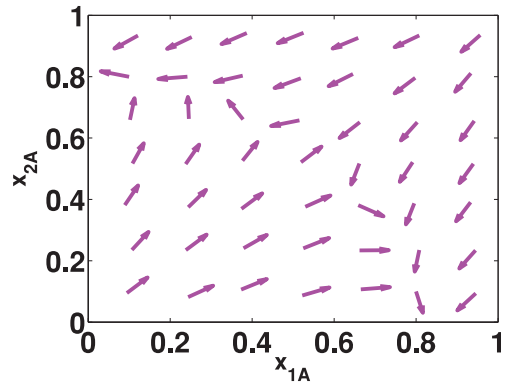

(a) $\alpha=0.3$

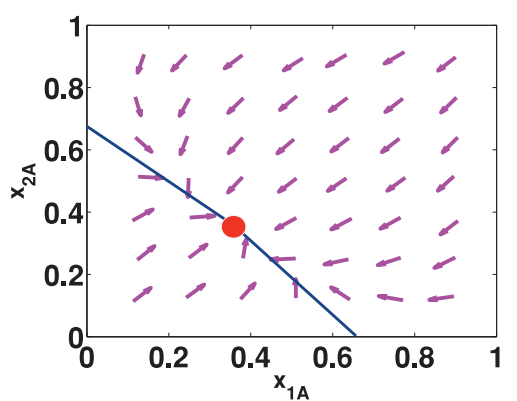

(b) $\alpha=0.7$

Figure $4 \mid$ Phase portraits of Eq. (6) under weak selection $(\boldsymbol{\beta}=\mathbf{0 . 0 1})$. The direction of the velocity field is indicated by arrows. We set $N_{1}=150, N_{2}=100$, $L_{1}=6, L_{2}=9, \omega=0.01$. When $\frac{a}{k_{A A}}+\varepsilon<\frac{c}{k_{A B}}$ and $\frac{b}{k_{A B}}>\frac{d}{k_{B B}}+\varepsilon\left(a=1.5, b=1, c=3, d=0, k_{A A}=0.6, k_{A B}=0.4\right.$ and $\left.k_{B B}=0.8\right)$, we show two cases of phase portraits when the intra-group bias $\alpha$ changes. (a) For $\alpha<\alpha^{*}=0.65(\alpha=0.3)$, the velocity field diverges to the corners near $(0,1)$ and $(1,0)$, which indicates that system (6) cannot reach an asymptotically stable consensus state. (b) For $\alpha>\alpha^{*}=0.65$ ( $\alpha=0.7$ ), the velocity field converges to the interior equilibrium, which means that system (6) reaches an asymptotically stable consensus at the interior equilibrium.

interior equilibrium, but also enhances the speed of reaching consensus (See Fig. 5(c) and (d)). Note that the eigenvalues of the Jacobian matrix at $E_{3}$ are $\lambda_{1}=-4 \kappa c_{5}\left(1+\frac{\alpha c_{5}}{c_{1}}\right)$ and $\lambda_{2}=-2 \kappa c_{5}\left(1+\frac{\alpha c_{5}}{c_{1}}\right)(2 \alpha-1)-\frac{1}{4}$, respectively. Thus, increasing $\alpha$ means decreasing the eigenvalues of $\lambda_{2}$, which indicates that system (6) reaches the asymptotically stable consensus state at a higher speed.

Case 3: Consensus of the bistable-type game.

When $\frac{a}{k_{A A}}>\frac{c}{k_{A B}}, \frac{b}{k_{A B}}<\frac{d}{k_{B B}}$, and $\alpha>\alpha^{*}$, both $E_{1}$ and $E_{2}$ are stable, and $E_{3}$ is unstable. Since the final state converges to $E_{1}$ or $E_{2}$, system (6) in the whole population is all- $A$ or all- $B$, if $k_{A A}<$
$k_{A B}$ and $k_{B B}<k_{A B}$. This result indicates that all players are inclined to interact with the opponents of same strategies as themselves'.

Besides, when $\alpha<\alpha^{*}, E_{3}$ becomes a saddle-point, so the intragroup attaching bias $\alpha$ does not affect global stability of system (6). A representative model is the coordination game. In this case, strategies $A$ and $B$ are bistable in the interdependent populations, and system (6) reaches an asymptotically stable consensus state at $E_{1}$ or $E_{2}$ (See Fig. 6). Note that the equilibrium, to which the system converges, depends on the initial fraction of $A$ and the interior unstable equilibrium $\left(x_{1, A}^{*}, x_{2, A}^{*}\right)$, where $x_{1, A}^{*}=x_{2, A}^{*}=E_{3}^{*}$. If the initial condition $x_{i, A}(0)>x_{i, A}^{*}$, system (6) converges to all- $A$; otherwise, to all- $B$. The effects of initialization on the frequency of strategy $A$ in both groups are shown in Fig. 7. Therefore, when the interactions between players under the payoff matrix $M^{\prime}$ are of the bistable-type game, two inter-

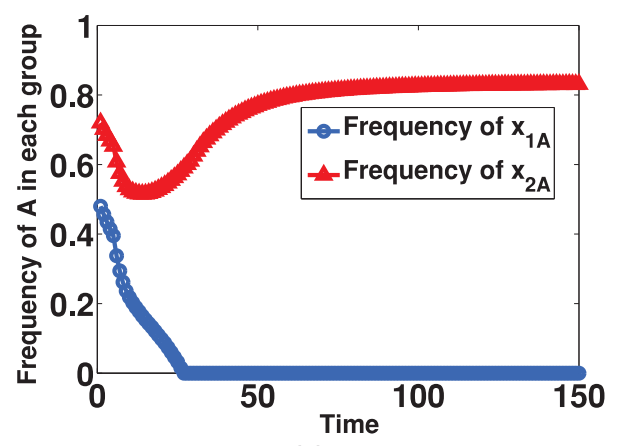

(a) $\alpha=0.3$

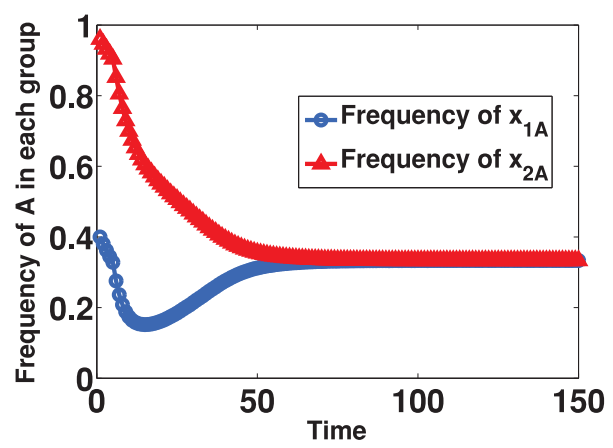

(c) $\alpha=0.6$

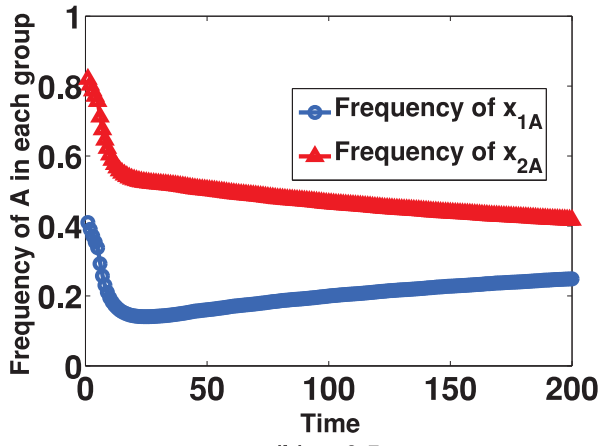

(b) $\alpha=0.5$

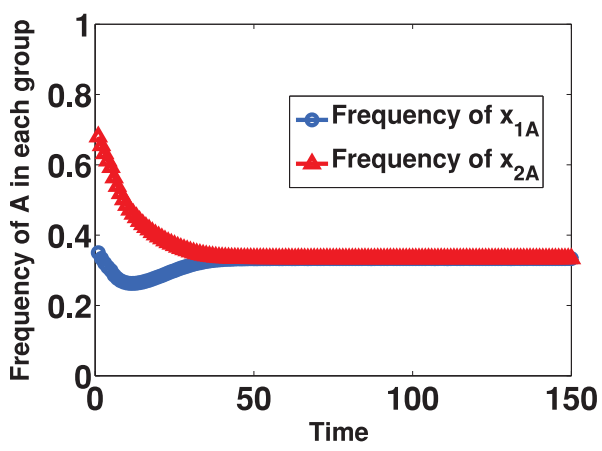

(d) $\alpha=0.7$

Figure 5 Simultaneous time-evolution of the frequency of strategy $A$ in Group-1 and Group-2 under weak selection $(\boldsymbol{\beta}=0.01)$. Initially, strategy $A$ is randomly distributed in Group-1 and Group-2. We set $N_{1}=150, N_{2}=100, L_{1}=6, L_{2}=9, \omega=0.01, a=1.5, b=1, c=3, d=0, k_{A A}=0.6$, $k_{A B}=0.4$ and $k_{B B}=0.8$. 


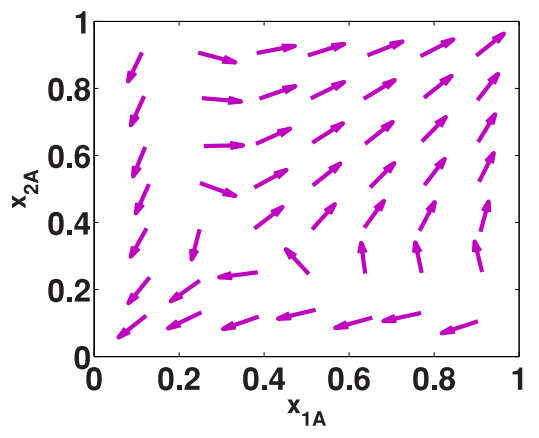

Figure $6 \mid$ Phase portrait of Eq. (6) under weak selection $(\boldsymbol{\beta}=0.01)$. The direction of the velocity field is indicated by arrows. We set $N_{1}=150, N_{2}=$ $100, L_{1}=6, L_{2}=9, \omega=0.01$. When $\frac{a}{k_{A A}}>\frac{c}{k_{A B}}, \frac{b}{k_{A B}}<\frac{d}{k_{B B}}$ and $\alpha>\alpha^{*}=$ $0.65\left(a=2, b=0, c=1.5, d=1, k_{A A}=0.4, k_{A B}=0.6, k_{B B}=0.8\right.$ and $\alpha=$ 0.7 ), the velocity field converges to the corner equilibrium $E_{1}$ or $E_{2}$, which means system (6) reaches an asymptotically stable consensus relying on the initialization.

active groups converge to a homogeneous consensus state of all- $A$, or all- $B$, which relies on the initialization.

Non-weak selection. For non-weak selection intensity, the perturbation analysis does not work. Besides, the results derived under weak selection fail to extend to strong selection intensity ${ }^{58,62,63}$. Therefore, we numerically simulate to illustrate the consensus state in interdependent populations. It is shown that: when the consensus state refers to the homogeneous population with only one strategy, the intensity of selection does not change the final convergence state (See Fig. 8(a), (b) and (d)); when the consensus state refers to the non-homogeneous population with coexistent strategy, it will loses its stability under non-weak selection, which drives the two populations to non-consensus states (See Fig. 8(c)). Intuitively, the homogeneous consensus is robust for perturbations, so that very strong selection can not force the consensus being extinct. While the non-homogeneous consensus states is relatively unstable, and sufficiently strong selection can force the consensus deviating from the previous consensus state. Therefore, the homogeneous consensus states in interdependent populations are robust in arbitrary selection intensity.

\section{Discussion}

To sum up, we have found that natural selection leads to stablized consensus, where the population structure is not only interdepend- ent but also dynamical. For neutral selection, we find that consensus can always be reached, yet none of these consensus states are stable. In fact, neutral selection will drive the population from one consensus state to another from time to time. When selection is present yet weak, the two interactive groups cannot always reach a consensus state. Interestingly, once the consensus is reached, it must be asymptotically stable. Therefore, natural selection here acts as a doubleedged sword: it may drive the two populations to non-consensus states, which never occurs when selection is absent; however, once consensus is established by selection, it is evolutionarily stable.

Importantly, the interdependent structure allows us to address how the intra-group attaching bias affects the stabilization of consensus. This intra-group bias is similar to the in-group bias where the structure is taken as a community. In particular, if all the individuals are only enabled to attach to individuals in the same group when adjusting their neighbourhood, i.e., under strong ingroup bias, the two interactive groups become isolated sooner or later. And each group degenerates to a non-interdependent population, which has been intensively discussed before ${ }^{15,16,34-37}$. On the other hand, allowing individuals to attach the other group yields two initially isolated groups connected. In this case, we find the intra-group attaching bias plays a major role in the convergent speed that the whole population reaches consensus.

It turns out that the consensus state strongly depends on i) what kind of conflicts they are engaging in, and ii) how players adjust their partnership. In fact, the neighbourhood adjusting rules transform the local competition to other types ${ }^{49,50}$. Interestingly, this transformation can be captured by the modified matrix Eq. (3). Specially, for the modified payoff matrix with all the entries equal to each other, the evolutionary process is independent of players' payoff. This scenario can emerge even when the local competition is frequency dependent, provided the neighberhood adjusting rule is appropriate. In this case, the interaction is similar to neutral selection, there is neither good nor bad strategies, and the conflicts among players degenerate to the voter model ${ }^{64,65}$. For the entry of the modified matrix Eq. (3) satisfy $\frac{a}{k_{A A}}>\frac{c}{k_{A B}}$ and $\frac{b}{k_{A B}}>\frac{d}{k_{B B}}+\varepsilon$, i.e., all players are apt to interact with strategy $A$ regardless of their own strategies, and the conflict in the interdependent populations become a dominant-type game. It is best to stick to dominant strategy no matter what other co-players perform, and the system always reaches a consensus of homogeneous state with the dominant strategy. Similarly, for the bistable-type game, the system reaches a consensus of a homogeneous state. And it is up to the initial fraction of strategy $A$ in each interactivegroup that which homogenous state it ends up with. In the case of the coexisting-type game, the system can reach a consensus of coexistent

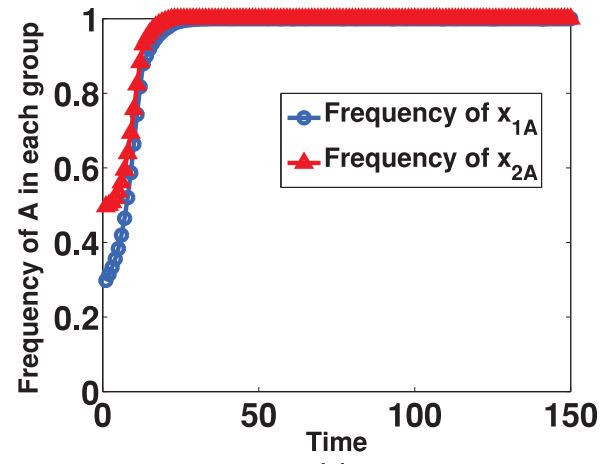

(a)

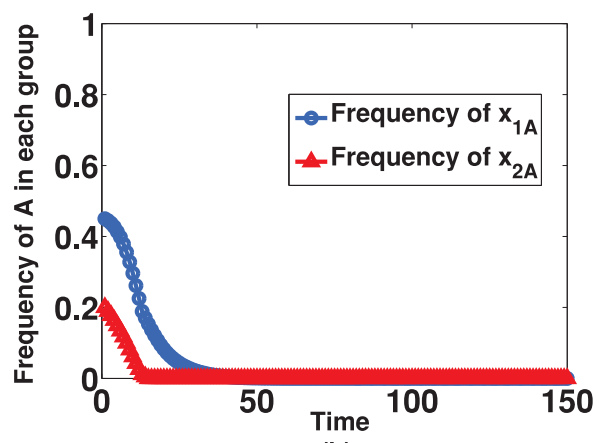

(b)

Figure $7 \mid$ Simultaneous time-evolution of the frequency of strategy $\boldsymbol{A}$ in Group-1 and Group-2 under weak selection $(\boldsymbol{\beta}=\mathbf{0 . 0 1})$. We set $N_{1}=150, N_{2}=$ $100, L_{1}=6, L_{2}=9, \omega=0.01, a=2, b=0, c=1.5, d=1, k_{A A}=0.4, k_{A B}=0.6, k_{B B}=0.8$. (a) When $x_{1, A}(0)=0.36>x_{1, A}^{*}=0.33$ and $x_{2, A}(0)=0.46>x_{2, A}^{*}=0.33$, system (6) reaches an asymptotically stable consensus state of all- $A$. (b) When $x_{1, A}(0)=0.39>x_{1, A}^{*}=0.33$ but $x_{2, A}(0)=0.22<x_{2, A}^{*}=0.33$, system (6) reaches an asymptotically stable consensus state of all- $B$. 


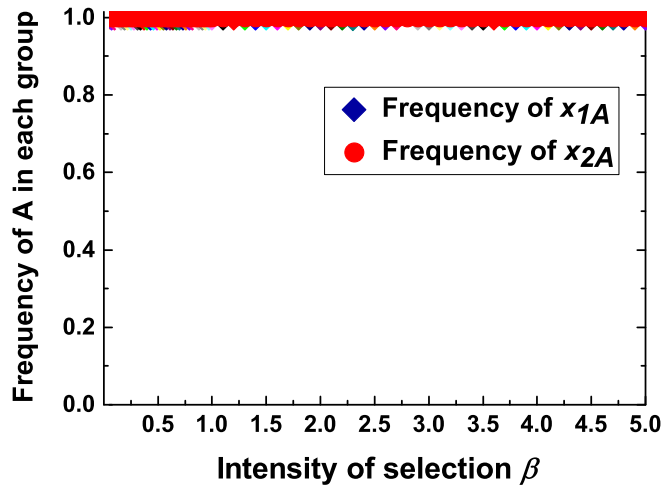

(a)

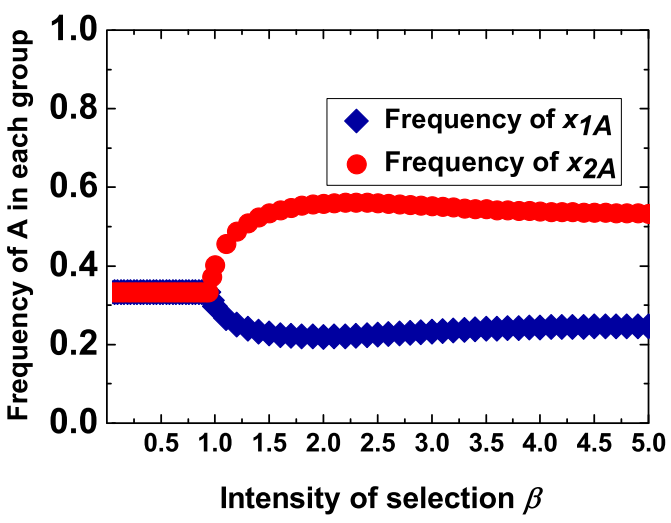

(c)

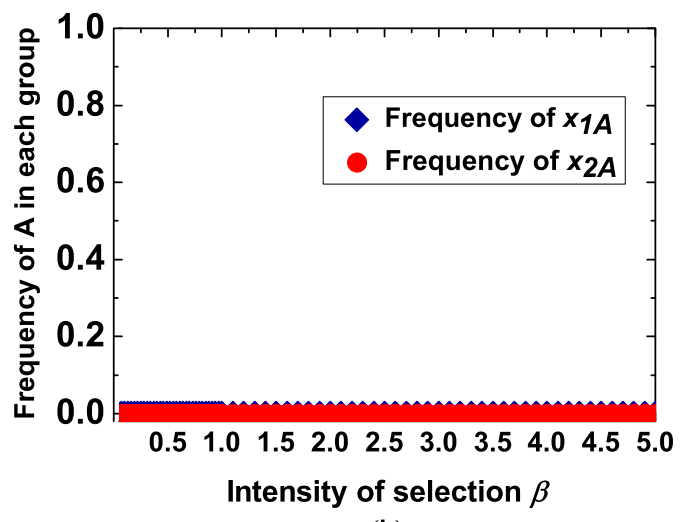

(b)

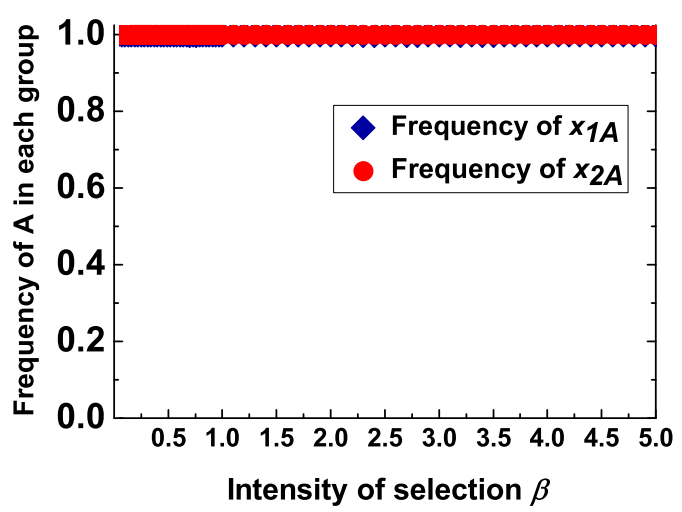

(d)

Figure $8 \mid$ The frequency of strategy in each groups under arbitrary selection intensity. Each data point is averaged over 50 independent runs, and in each run we set the time window as $1.5 \times 10^{4}$ generations to guarantee the final state converged. We set $N_{1}=150, N_{2}=100, L_{1}=6, L_{2}=9, \omega=0.01$. (a) $a=2, b=1, c=1.5, d=0, k_{A A}=0.4, k_{A B}=0.6$ and $k_{B B}=0.8$, system (4) reaches an asymptotically stable consensus state of all- $A$. (b) $a=1.5, b=0$, $c=2, d=1, k_{A A}=0.6, k_{A B}=0.4$ and $k_{B B}=0.8$, system (4) reaches an asymptotically stable consensus state of all- $B$. (c) $a=1.5, b=1, c=3, d=0$, $k_{A A}=0.6, k_{A B}=0.4, k_{B B}=0.8, \alpha=0.7$ and system (4) reaches an asymptotically stable consensus state when selection intensity is weak, which, however reaches a non-consensus state when selection is non-weak. (d) $a=2, b=0, c=1.5, d=1, k_{A A}=0.4, k_{A B}=0.6, k_{B B}=0.8$ and $\alpha=0.7$, system (4) reaches an asymptotically stable consensus of all- $A$.

state at an interior equilibrium, and strong intra-group attaching bias not only benefits the formation of an asymptotically stable consensus, but also enhances the speed of reaching consensus. Intuitively, consensus seems to be more likely to come into being, if two groups are more likely to interact with each other. In biology, this refers to frequent gene-flow between two niches. And it is believed that convergent evolution is more likely to occur, since the environments of the two niches are similar ${ }^{66,67}$. Yet, our results highlight the importance of both the interaction between two groups and the conflict therein.

As the first stage, we simply restrict our model to two interactive groups, which is the minimum interdependent populations. For general interdependent populations with more interactive groups, the microscopic rule and analyses still work, though it can be technically tedious. What's more, based on the microscopic rule, the proposed macro-dynamics governing the frequency of the strategy in each group stands within the fast partnership rewiring process ${ }^{49-52}$, which opens an avenue via the down-to-up way to address how such coevolutionary dynamics affects the collective behaviors in interdependent populations.

1. Morris, S. C. Life's solution: inevitable humans in a lonely universe (Cambridge University Press, Cambridge, UK, 2003).
2. Grant, P. R., Grant, B. R., Markert, J. A., Keller, L. F. \& Petren, K. Convergent evolution of Darwin's finches caused by introgressive hybridization and selection. Evolution 58, 1588-1599 (2004).

3. Reynolds, C. W. Flocks, herds, and schools: A distributed behavioral model. Comput. Graph 21, 25-34 (1987).

4. Olfati-Saber, R. Flocking for multi-agent dynamic systems: algorithms and theory. IEEE Trans. Autom. Control 51, 401-420 (2007).

5. Castellano, C., Loreto, V., Barrat, A., Cecconi, F. \& Parisi, D. Comparison of voter and Glauber ordering dynamics on networks. Phys. Rev. E 71, 066107 (2005).

6. Gil, S. \& Zanette, D. H. Coevolution of agents and networks: Opinion spreading and community disconnection. Phys. Lett. A 356, 89-94 (2006).

7. Olfati-Saber, R. \& Murray, R. M. Consensus problems in networks of agents with switching topology and time-delays. IEEE Trans. Autom. Control 49, 1520-1533 (2004).

8. Ren, W. \& Beard, R. W. Consensus seeking in multiagent systems under dynamically changing interaction topologies. IEEE Trans. Autom. Control 50, 655-661 (2005).

9. Xiao, F. \& Wang, L. Consensus protocols for discrete-time multi-agent systems with time-varying delays. Automatica 44, 2577-2580 (2008).

10. Vicsek, T., Czirók, A., Ben-Jacob, E., Cohen, I. \& Shochet, O. Novel Type of Phase Transition in a System of Self-Driven Particles. Phys. Rev. Lett. 75, 1226-1229 (1995).

11. Lin, Z., Francis, B. \& Maggiore, M. State agreement for continuoustime coupled nonlinear systems. SIAM J Control 46, 288-307 (2007).

12. Zhan, J. Y. \& Li, X. Consensus of sampled-data multi-agent networking systems via model predictive control. Automatica 49, 2502-2507 (2013).

13. Conradt, L. \& Roper, T. J. Consensus decision making in animals. Trends. Ecol. Evol. 20, 449-456 (2005).

14. Vazquez, F., Eguíluz, V. M. \& San Miguel, M. Generic Absorbing Transition in Coevolution Dynamics. Phys. Rev. Lett. 100, 108702 (2008). 
15. Couzin, I. D. et al. Uninformed Individuals Promote Democratic Consensus in Animal Groups. Science 334, 1578-1580 (2011).

16. Sood, V., Antal, T. \& Redner, S. Voter models on heterogeneous networks. Phys. Rev. E 77, 041121 (2008).

17. Vespignani, A. Complex networks: The fragility of interdependency. Nature 464, 984-985 (2010).

18. Parshani, R., Buldyrev, S. V. \& Havlin, S. Critical effect of dependency groups on the function of networks. Proc. Natl. Acad. Sci. USA 108, 1007-1010 (2010).

19. Aguirre, J., Papo, D. \& Buldú, J. M. Successful strategies for competing networks. Nat. Phys. 9, 230-234 (2013).

20. Wang, Z., Szolnoki, A. \& Perc, M. Evolution of public cooperation on interdependent networks: the impact of biased utility functions. Europhys. Lett. 97, 48001 (2012)

21. Wang, Z., Szolnoki, A. \& Perc, M. Optimal interdependence between networks for the evolution of cooperation. Sci. Rep. 3, 2470 (2013).

22. Gómez-Gardeñes, J., Gracia-Lázaro, C., Floría, L. M. \& Moreno, Y. Evolutionary dynamics on interdependent populations. Phys. Rev. E 86, 056113 (2012).

23. Jiang, L. L. \& Perc, M. Spreading of cooperative behaviour across interdependent groups. Sci. Rep. 3, 2483 (2013).

24. Wang, Z., Szolnoki, A. \& Perc, M. Interdependent network reciprocity in evolutionary games. Sci. Rep. 3, 1183 (2013).

25. Jin, Q., Wang, L., Xia, C. Y. \& Wang, Z. Spontaneous Symmetry Breaking in Interdependent Networked Game. Sci. Rep. 4, 4095 (2014).

26. Radicchi, F. \& Arenas, A. Abrupt transition in the structural formation of interconnected networks. Nat. Phys. 9, 717-720 (2013).

27. Tang, C. B., Wang, Z. \& Li, X. Moderate intra-group bias maximizes cooperation on interdependent populations. PLOS ONE 9, e88412 (2014).

28. Lambiotte, R. \& Ausloos, M. Coexistence of opposite opinions in a network with communities. J. Stat. Mech. Theor. E 08, p08026 (2007).

29. Efferson, C., Lalive, R. \& Fehr, E. The coevolution of cultural groups and ingroup favoritism. Nature 321, 1844-1849 (2008).

30. Fu, F., Tarnita, C. E., Christakis, N. A., Wang, L., Rand, D. G. \& Nowak, M. A. Evolution of in-group favoritism. Sci. Rep. 2, 460 (2012).

31. Richardson, T. O., Perony, N., Tessonea, C. J. et al. Dynamical coupling during collective animal motion. arXiv:1311.1417 (2013).

32. Bergstrom, T. C. Evolution of Social Behavior: Individual and Group Selection. J. Econ. Perspect. 16, 67-88 (2002).

33. Wang, J., Wu, B., Wang, L. \& Fu, F. Consensus of population systems with community structures. Phys. Rev. E 78, 051923 (2009).

34. Suchecki, K., Eguíluz, V. M. \& San Miguel, M. Voter model dynamics in complex networks: Role of dimensionality, disorder, and degree distribution. Phys. Rev. E 72, 036132 (2005).

35. Fu, F. \& Wang, L. Coevolutionary dynamics of opinions and networks: From diversity to uniformity. Phys. Rev. E 78, 016104 (2008).

36. Yang, H. X., Wu, Z. X., Zhou, C. S., Zhou, T. \& Wang, B. W. Effects of social diversity on the emergence of global consensus in opinion dynamics. Phys. Rev. E 80, 046108 (2009)

37. Vilone, D., Ramasco, J. J., Sánchez, A. \& San Miguel, M. Social and strategic imitation: the way to consensus. Sci. Rep. 2, 686 (2012).

38. Castellano, C., Fortunato, S. \& Loreto, V. Statistical physics of social dynamics Rev. Mod. Phys. 81, 591-596 (2009).

39. Wang, Z., Liu, Y., Wang, L., Zhang, Y. \& Wang, Z. Freezing period strongly impacts the emergence of a global consensus in the voter model. Sci. Rep. 4, 3597 (2014).

40. Dobzhansky, T. Nothing in Glycobiology Makes Sense, except in the Light of Evolution. Am. Biol. Teach. 35, 125-129 (1973).

41. Zimmermann, M. G. \& Eguluz, V. M. Cooperation, social networks, and the emergence of leadership in a prisoner's dilemma with adaptive local interactions. Phys. Rev. E 72, 056118 (2005).

42. Segbroeck, S. V., Santos, F. C., Lenaerts, T. \& Pacheco, J. M. Reacting differently to adverse ties promotes cooperation in social networks. Phy. Rev. Lett. 102, 058105 (2009).

43. Santos, F. C., Pacheco, J. M. \& Lenaerts, T. Cooperation prevails when individuals adjust their social ties. PLoS Comput. Biol. 2, 1284-1291 (2006).

44. Gross, T. \& Blasius, B. Adaptive coevolutionary networks-a review. J. R. Soc. Interface 5, 259-271 (2008).

45. Perc, M. \& Szolnoki, A. Coevolutionary games-a mini review. Biosystems 99, 109-125 (2010).

46. Gross, T. \& Sayama, H. Adaptive Networks: Theory, Models and Applications Understanding Complex Systems (Springer-Verlag, New York, 2009).

47. Blume, L. E. The statistical mechanics of strategic interaction. Games Econ. Behav. 5, 387-424 (1993).

48. Szabó, G. \& Tőke, C. Evolutionary prisoner's dilemma game on a square lattice. Phys. Rev. E 58, 69-73 (1998).
49. Wu, B., Zhou, D., Fu, F., Luo, Q., Wang, L. \& Traulsen, A. Evolution of Cooperation on Stochastic Dynamical Networks. PLoS ONE 5, e11187 (2010).

50. Wu, B., Zhou, D. \& Wang, L. Evolutionary dynamics on stochastic evolving networks for multiple-strategy games. Phys. Rev. E 84, 046111 (2011).

51. Pacheco, J. M., Traulsen, A. \& Nowak, M. A. Coevolution of Strategy and Structure in Complex Networks with Dynamical Linking. Phys. Rev. Lett. 97, 258103 (2006).

52. Pacheco, J. M., Traulsen, A. \& Nowak, M. A. Active linking in evolutionary games. I. Theor. Biol. 243, 437-443 (2006).

53. Taylor, C. \& Nowak, M. A. Transforming the dilemma. Evolution 61, 2281-2292 (2007).

54. Traulsen, A., Claussen, J. C. \& Hauert, C. Coevolutionary Dynamics: From Finite to Infinite Populations. Phys. Rev. Lett. 95, 238701 (2005).

55. Wu, B., Altrock, P. M., Wang, L. \& Traulsen, A. Universality of weak selection. Phys. Rev. E 82, 046106 (2010).

56. Nowak, M. A., Sasaki, A., Taylor, C. \& Fudenberg, D. Emergence of cooperation and evolutionary stability in finite populations. Nature 428, 646-650 (2004).

57. Ohtsuki, H., Hauert, C., Lieberman, E. \& Nowak, M. A. A simple rule for the evolution of cooperation on graphs and social networks. Nature 441, 502-505 (2006).

58. Wu, B., García, J., Hauert, C. \& Traulsen, A. Extrapolating weak selection in evolutionary games. PLoS Comput. Biol. 9, e1003381 (2013).

59. Charlesworth, J. \& Eyre-Walker, A. The other side of the nearly neutral theory, evidence of slightly advantageous back-mutations. Proc. Natl. Acad. Sci. USA 104, 16992-16997 (2007).

60. Akashi, H. Inferring weak selection from patterns of polymorphism and divergence at "silent" sites in drosophila dna. Genetics 139, 1067-1076 (1995).

61. Traulsen, A., Semmann, D., Sommerfeld, R. D., Krambeck, H. J. \& Milinski, M. Human strategy updating in evolutionary games. Proc. Natl. Acad. Sci. USA 107, 2962-2966 (2010).

62. Fudenberg, D., Nowak, M. A., Taylor, C. \& Imhof, L. Evolutionary game dynamics in finite populations with strong selection and weak mutation. Theor. Popul. Biol. 70, 352-363 (2006)

63. Traulsen, A., Shoresh, N. \& Nowak, M. A. Analytical results for individual and group selection of any intensity. Bull. Math. Biol. 70, 1410-1424 (2008).

64. Sood, V. \& Redner, S. Voter model on heterogeneous graphs. Phy. Rev. Lett. 94 178701 (2005)

65. Mobilia, M., Petersen, A. \& Redner, S. On the role of zealotry in the voter model. J. Stat. Mech. Theor. E 8, p08029 (2007)

66. Petit, R. J. \& Excoffier, L. Gene flow and species delimitation. Trends. Ecol. Evol. 24, 386-393 (2009)

67. Elmer, K. R. \& Meyer, A. Adaptation in the age of ecological genomics: insights from parallelism and convergence. Trends. Ecol. Evol. 26, 298-306 (2011).

\section{Acknowledgments}

This work was partly supported by the National Key Basic Research and Development Program (No. 2010CB731403), the National Natural Science Foundation (No. 61273223), the Research Fund for the Doctoral Program of Higher Education (No. 20120071110029) and the Key Project of National Social Science Fund (No. 12\&ZD18) of China. C.B.T. also acknowledges the financial support by the Academic New Artist Ministry of Education Doctoral Post Graduate (No. JFH1232003) of China. B.W. acknowledges the financial support from the Max Planck Society.

\section{Author contributions}

C.B.T. and B.W. contributed to this work equally. C.B.T., B.W. and X.L. planned the study; C.B.T., B.W. and J.B.W. performed the experiments, analyzed the data, and prepared the figures; C.B.T., B.W. and X.L. wrote the paper.

\section{Additional information}

Supplementary information accompanies this paper at http://www.nature.com/ scientificreports

Competing financial interests: The authors declare no competing financial interests.

How to cite this article: Tang, C.-B., Wu, B., Wang, J.-B. \& Li, X. Evolutionary Origin of Asymptotically Stable Consensus. Sci. Rep. 4, 4590; DOI:10.1038/srep04590 (2014).

This work is licensed under a Creative Commons Attribution-NonCommercialNoDerivs 3.0 Unported License. The images in this article are included in the article's Creative Commons license, unless indicated otherwise in the image credit; if the image is not included under the Creative Commons license, users will need to obtain permission from the license holder in order to reproduce the image. To view a copy of this license, visit http://creativecommons.org/licenses/by-nc-nd/3.0/ 